the controversial "hot spots" theory, and suggest that the Philippine Ridge (by then extinct) may not have changed its motion to the same extent. According to the authors this would produce the interesting situation in which a transform fault becomes a subduction zone. It is also interesting to note that this zone is continued to the south beyond the Philippine Ridge. These postulated movements of the Pacific Plate must be examined to see how they relate to the magnetic lineations in the West Pacific. During the Eocene ( 40 to 60 million years ago) Uyeda and Ben-Avraham suggest that the transform fault became an island arc, which, 40 million years ago, split into two by the Karig process to form the Parece-Vela Basin. From then on Karig's crustal extension model explains the formation of the Parece-Vela Basin and Mariana Island Arc system.--C. M. R. F.

\section{Steady State Obituary ?}

FOR more than fifteen years after its invention in 1948, the steady state cosmology of Bondi, Gold and Hoyle exercised a strong hold on the imaginations of British astronomers, and probably an even stronger hold on the imaginations of those members of the wider population with a philosophical turn of mind. Those secularists who were unsatisfied by the discomfiture of established religion by Copernicus, Galileo and Darwin presumably rejoiced at the abolition of the Creation altogether. The absence of an end of the world, a matter inconveniently undecided by conventional big-bang cosmology, was obviously of benefit to all. The Universe was to continue for ever to present the same face to us, the recession of the galaxies compensated by the formation of new ones from continuously created matter. The fact that at that time the age of the Universe according to the bigbang picture seemed to be very much shorter than the age of the Galaxy provided additional support for those for whom philosophical arguments are insufficient.

Outside Britain the theory had fewer converts, although whether this was due to conservatism, or to inconsistency with the Book of Genesis or Dialectical Materialism, is unclear. Even in Britain the theory had its enemies. One radio observatory was rumoured to have decided to commit its entire scientific effort to the destruction of the theory. Certainly it was radio source counts that provided the first stumbling block. As the Universe is to be the same at all places and all times, one has only to study the properties of a sample of it in order to be able to predict the behaviour of the rest. In a recent issue of Nature $(\mathbf{2 4 0}, 399$; 1972) M. Schmidt uses as his sample a set of twenty-six completely identified sources brighter than 30 flux units at a frequency of $178 \mathrm{MHz}$ to predict the number of sources expected in the steady state theory at fainter flux levels. The number actually observed is greater than the steady state prediction by a large factor which depends on how far away the quasars are presumed to be.

Inconsistency of the steady state cosmology with radio source counts was claimed by Ryle and Clarke back in 1961, before quasars had been discovered. Hoyle and Narlikar responded with an extremely ingenious modification of the steady state idea (Mon. Not. Roy. Astron. Soc., 123,$133 ; 1961)$. The continuous creation required by the theory was to occur in the form of massive (about $10^{5}$ galaxies)) discrete primary condensations at irregular intervals, instead of taking place at a uniform rate at all places and all times. Within one of these condensations an expanding system of objects all of the same age would be seen. If the probability of a galaxy being a radio source depends on age, an apparent evolutionary effect would be seen within the condensation. The Universe is in a steady state on average, but coherent temporal fluctuations occur over extended regions.

Although this modified steady state theory has been for the most part ignored by supporters of the big-bang, it is probably fair to say that the modification was never fully developed by its proponents. Of course, the severe simplicity of the original idea has been lost. Critics argue that it is necessary for one to be in rather a special place, near the centre of one of these condensations, in order to avoid strong anisotropy in the distribution of radio sources. The distribution of galaxies brighter than $16 \mathrm{mag}$ is, however, not especially isotropic, and it would be surprising if radio sources do not ultimately reflect this anisotropy.

A more serious difficulty for the steady state theory was the discovery of microwave background radiation by Penzias and Wilson in 1965. Apart from some as yet unresolved anomalies in the millimetre waveband, this radiation has the spectrum of a $2.7 \mathrm{~K}$ black body and is exceedingly isotropic, consistent with being a relic of the fireball phase of the big-bang. Pace the radio astronomers, it is this microwave background radiation which has turned most steady state supporters into big-bang bigots.

By 1969 Hoyle had shifted to much weaker ground. In his Bakerian lecture (Proc. Roy. Soc. A, 308, 1; 1969) he suggested that the source counts at faint flux levels fit the steady state predictions well: all that is wrong is a deficiency of four or five sources per steradian at high flux levels. This figure was based on back-of-envelope considerations of the unidentified sources in the $3 \mathrm{C}$ survey. On the basis of a more detailed calculation, Maarten Schmidt argues in his report that his sample of twenty-six sources would in fact have to be deficient by a factor of five, far too much for a statistical fluctuation.

Whether the redshifts of quasars are entirely cosmological is still the subject of hot debate, as may be seen from the many articles on the subject in Nature during the past year. Schmidt concludes that "whatever is the interpretation of the quasar redshifts, the observed radio source counts, identifications and redshifts are incompatible with the steady state cosmology". This is true provided the quasars in his sample are typical representatives of a population of objects spread uniformly through space. But if, first, all quasars belong to a local irregularity; second, most unidentified sources are quasars; third, many identifications of radio sources with galaxies fainter than $18 \mathrm{mag}$ are mistaken (otherwise the distribution of radio galaxies alone is inconsistent with the steady state); and, fourth, the microwave background is not cosmic black body radiation but is, for example, due to starlight reradiated by dust (Hoyle drew attention in his Bakerian lecture to the similarity of the energy densities involved), then even the strict steady state cosmology could perhaps be saved.

The strong arguments that can be mounted against all four of these conditions seem, however, entirely convincing.-M. R.-R. 